\section{Health informatics 3.0}

\author{
Dipak Kalra \\ University College London
}

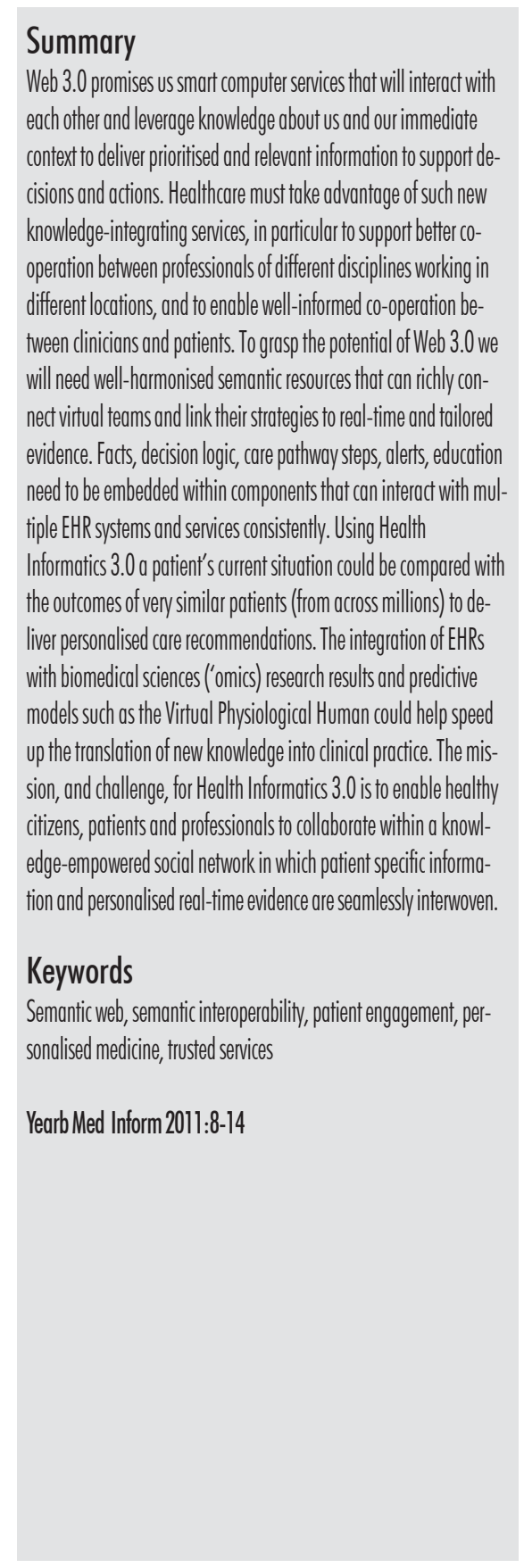

It's time for health informatics to evolve from enabling the information society to creating the knowledge society: health informatics 3.0 .

The vision of the semantic web (a critical element of Web 3.0) is of information objects that are richly indexed by knowledge models and bound to collaborating components within a distributed computing ecosystem [1], bundled as smart services that interact with each other to deliver personalised (tailored, relevant and prioritised) information that supports the decisions and actions of individuals and communities. Services will collaborate to deliver an integrated and holistic solution to its users. In other words, individuals will no longer utilise computers to search for and locate specific items of information to digest and weight up, but will pose problems and receive answers to questions like:

- What are my best care management options for this particular patient?

- What will happen to renal function if I discontinue this drug?

- What is the optimal dose in this patient to maximise effectiveness and minimise adverse effects?

- How much should we budget for children's cancer services next year?

Web 3.0 computers will work out what information they need, where to get it from and how to access it. They will filter and prioritise and personalise what they find, and present the user with a polished set of reasoned options from which to make a final choice. Smart services will present individuals with information when they need it and in the formats (modality, device, level of detail) that are best suited to that individual at that time. This is indeed a grand vision!

Healthcare now needs us to provide such knowledge-integrating and collaborating services: not as prototypes and pilots, but on a wide scale.

Increasing sophistication of care, with an ageing population [2] and more and more illnesses becoming long-term conditions (such as HIV [3]), requires greater co-operation between professionals of different disciplines working in different locations, knowing each others care goals, progress made and any difficulties encountered. This needs more than sharing the record of what has been done (EHRs), but sharing each other's clinical care strategy, options and logistic constraints, to determine optimal ways of aligning efforts. We accomplish this best today by discussing patients in team meetings. Physical (or online) meetings could only cover a minority of patients, and team collaboration needs a way of scaling up virtually, through knowledge services.

The rapid pace of bio-science discovery, the advent of personalised therapies, and the overwhelming volume of new clinical evidence, combined with demands for safe, high quality and equitable standards of care, has already encouraged professionals to replace historic sources of knowledge (textbook plus apprenticeship plus anecdote) with evidence based care. The access to evidence has itself evolved from individuals searching the literature (with greater or lesser skills at 
critical appraisal) to peer-developed guidelines that have filtered and combined published evidence into a specification of best practice $(50+$ pages each!), and now progressively being mapped into clinical workflows (as care pathways) to make them more usable. But we need to advance from these forms of evidence (which are ultimately informed by large expensive studies that take years to deliver results) to empirical data integrated across large populations, collected systematically and therefore comparable: real time outcomes.

Individualised evidence would take this a step further, in which a patient's current situation could be compared with the patterns and outcomes of very similar patients (from across millions) in order to deliver point of care recommendations that are highly relevant. Such case based systems already exist, but are usually focused on a single condition, intensively curated and only within one (innovative) hospital. Linking case based analyses to emergent knowledge could radically shorten the "bench to bedside" translation from over a decade to months. Such capability should be available to inform the routine care of all patients, whenever needed.

Most of our current guidelines are for single diseases, but patients rarely have only one. The most realistic way of building up an understanding of how diseases and treatments interact, and the optimal ways to manage multiple conditions, is to study (data mine) large-scale EHR repositories. European level disease specific repositories are already proving useful for knowledge discovery (e.g. [4]), but these are expensive to create and maintain. Large population repositories (ideally multi-national) are now needed. This will require health informatics to develop scalable and convincing solutions for privacy protection, de-identification, informationknowledge integration and the real-time analysis of distributed repositories, and friendly ways of presenting this knowledge to busy clinicians.
Much of health informatics, and also the needs of health care, have focussed up to now on managing collections of data: for example improving interfaces and devices for data capture, information models and databases to store and process the data, interoperability standards to communicate data and allow us to grow longitudinal health records, single condition guidelines and simple decision support, analysis of reporting data sets and data warehouses, and a little bit of near patient tele-care. The interpretation of complex images and signals has perhaps had the greatest real-world impact so far. Bioinformatics is bringing us fresh ways to consider diseases and treatments, but we need to improve its integration with routine health care (beyond inherited disorders and chemotherapy).

Health IT has so far helped us to cope with the increasing data volumes, but as we finally succeed in joining up disjointed parts of our health system how will we avoid information overload? Will our clinicians and our managers simply get more data, or will they have useful information presented in ways that can be applied efficiently and effectively? Will they spend more time sifting through health information and published knowledge, or less? Will service management decisions (e.g. commissioning) be more wisely informed or remain simplistic but backed by larger volumes of data, as poor in quality as today?

Next generation health care poses some quite powerful challenges that require us to harness and exploit knowledge at a much greater scale than we presently can. To grasp the potential of Web 3.0, we need to develop wellharmonised semantic resources that can richly connect virtual teams and link their strategies to real-time evidence to enable better, safer and more efficient care. We need to accelerate new research and leverage better the value of bio-informatics research. We need to engage and empower patients and healthy citizens in personalised healthcare.

\section{Harmonised Semantic Resources}

The cornerstone of health informatics 3.0, of collaborating components and smart services, is knowledge integration. Yet, today, our knowledge representations across healthcare, clinical research and bio-medicine are limited, and poorly interoperable.

Investments in health informatics research, standards, product development and deployment are gradually enabling the integration of data within and between organisations. This is known colloquially as syntactic interoperability: the ability to exchange data, but only being able to use it meaningfully if parties have agreed in advance on how they will represent meaning within the data. In the hierarchy of semantic interoperability levels defined in the SemanticHEALTH Report [5], Web 3.0 services will require Level 3 interoperability: full semantic inter-operability with sharable context and seamless co-operability (i.e. received data can be combined seamlessly with local data and processed homogeneously).

We need guideline and decision support systems, notification and alerting components, and analytic tools to be able to consistently process integrated health data drawn from multiple EHR systems. New generation personalised medicine needs the integration of EHRs with biomedical sciences ('omics) research results and predictive models such as the Virtual Physiological Human. Clinical and public health research, and clinical trials, need to leverage the value locked in our existing EHRs. The knowledge representations that underpin each of these sub-disciplines need to be formalised and aligned.

Important progress has been made in this direction, and there are some early successes. We have known for many years that prescribing decision support systems can help prevent serious error, and that they require access 
to comprehensive allergy, diagnosis and past medication data from the EHR in a processable form, cross-mapped to data on drugs, their effects and active ingredients. Such decision support has been shown long ago to work consistently across vendor products [6]. To provide this level of interoperability on a wide scale, the meaning of the clinical information within EHRs needs to be formally and consistently represented in order to be understood computationally and re-used by other systems whose information models are different, and be mapped to a wide range of knowledge resources (such as conditional criteria within guidelines, eligibility criteria in trial protocols, alerting criteria in surveillance systems).

Achieving such interoperability across the breadth of health and healthcare is the challenge that needs urgently to be addressed. It is hard to believe that the origins of the EHR archetype are now almost 15 years old [7]. This was conceived as the most basic level of knowledge representation for the EHR: standardised clinical models to provide a common representation to which data from heterogeneous systems can be mapped, to support harmonised integration and consistent analysis. The need to build up a library of clinical content of this kind has been accepted the world over, but the value of authoring this knowledge using a compatible approach (archetypes, or something else) and some degree of cross-specialty coherence seems to have escaped attention. Tens of countries have each invested millions each in building up patchy, inconsistent and incompatible collections of clinical models, some bound to terminology, probably of variable quality (since validation is nearly non-existent) and with limited uptake within products. Yet, in 1996, this was only envisaged as the starting block, not the holy grail!

The challenge ahead is substantial. Useful semantic interoperability hinges upon widespread and dependable access to published and maintained col- lections of coherent and quality-assured semantic resources: clinical models such as archetypes and templates, mapped to EHR interoperability standards and bound to well specified multilingual terminology value sets, indexed and correlated with each other via ontologies and referenced from modular care pathway components. Similarly, libraries of interoperable rules and queries to support decisionmaking, to generate alerts and to represent the steps within care pathways, need to be built up and adopted consistently, so that services that use them behave uniformly and predictably. For example, a multi-disease care pathway should be able to seamlessly combine the parts of single-disease pathways without inconsistency or duplication. An antibiotic prescribing system should be able to check a patient's documented health status including renal and liver function, pregnancy status, allergies, recent antibiotic prescriptions, the relevant clinical guideline, the outcome of other patients recently prescribed antibiotics in that hospital for the same indication, local and regional resistance patterns and the hospital's preferred formulary in order to present the prescriber with a prioritised short-list of options that are all known to be safe and effective [8].

Multi-lingual resources are needed to support cross-border care and to enable cross-border aggregation for research and population health management. Multi-jargon resources are also needed so that users of differing levels of expertise can review and understand health information and knowledge - for example a specialist and patient sharing the same information but viewing it differently.

Generating semantically coherent resources requires strengthening the alignment of the various standards development organisations (SDOs) and investing in harmonising the artefacts they produce. Health informatics standards development has historically been piecemeal: not just between SDOs (many of which are nowadays collaborating through the Joint Initiative Council) but more importantly between individual standards. These are still usually developed, balloted and published in isolation. Yet few informatics solutions rely upon only one standard: too little effort is invested in working out and validating a solution-oriented portfolio of standards, and in delivering coherent guidance to adopters (industry, purchasers and users). IHE is making a contribution, especially for DICOM standards, but solution orientation must be designed into standards from the outset, not worked out as a best fit afterwards. The terminology-record structure binding issue is a classical example of a cumbersome solution to a largely preventable problem [9]. SDOs need to shift their processes towards ensuring industry and user relevance, appropriate levels of sophistication and complexity that are affordable and so can be readily taken up by markets, promoting and supporting adoption, and learning from experience. Many SDOs do almost none of this today. Successful alignment will also depend upon the willingness of each SDO to cede certain areas of scope in favour of standards developed by other SDOs.

We have limited global experience of developing harmonised collections (libraries) of semantic resources of sufficient scale. There is a paucity of examples of good practice in how semantic resources should be defined, validated and widely accepted, how users should be trained to improve the quality and consistency of EHR documentation, and how to best enable patients and healthy citizens to become knowledge-empowered participants in maintaining good health and managing illness.

It is high time for a multistakeholder international initiative to build up this high quality clinical content, through a formalised, coordinated and well-governed process. Experts at a recent ARGOS project workshop in Washington [10] agreed that semantic interoperability is too 
complex to be tackled right across health care at once. Attention should first be on areas of practice that can leverage existing clinical consensus and accepted evidence, for example chronic diseases such as heart failure, population health challenges such as childhood obesity. Even within such a targeted strategy, not all clinical data needs to be semantically processed. Priority should be given to the data that have known computational value i.e. for which there is a knowledge related exploitation, for example care pathway support or patient safety. Research is needed on the criteria that help to determine what parts of, and how much of, a health record is useful to structure and code to achieve Level 3 interoperability.

Starting with priority areas does not imply that a piecemeal solution will work: semantic interoperability is a holistic problem, and needs to be addressed through a coherent strategy. The starting points need to be clearly positioned on a roadmap towards richer inter-operability, and the approaches scalable to more complete semantic inter-operability as an evolutionary process.

It is probably fair to say that formal knowledge representation (ontology) is still primarily seen as a research capability in health informatics rather than ready to scale. Yet ontology will be vital as the means to broker across different knowledge representations, between models of meaning and models of use [11], between different communities such as bio-science research, population health research and clinical practice, between service providers and service planers. Each of these stakeholders needs their own view of the knowledge space. This is not solved by one or a few giant ontologies, but by focussed ontological services that are each fit for a dedicated stakeholder group and purpose - at the appropriate level of detail and coverage - yet are able to collaborate with others.

Growing this body of harmonised semantic resources should now be a high priority for health informatics, clinical professional bodies and eHealth programmes.

The benefits of semantic inter-operability occur some way downstream of the investments needed in tools, clinical content and in knowledgedriven systems. The lack of clear business models to justify these medium to long term investments has probably been a significant barrier to progress to date, and an alignment of value propositions is needed for this effort to progress and scale up. This in turn requires inter-governmental strategic co-operation.

\section{Accelerate and Leverage Knowledge Discovery}

The present gold standard for new clinical evidence is a clinical trial or a population health (cohort) study: both of these are expensive to undertake and may take years to deliver results. Published study results that could impact on strategic clinical decisions then take further years to influence routine clinical practice on a wide scale. This "bench to bedside" delay of up to a decade prolongs the use of potentially inefficient and ineffective practice and risks preventable harm to patients. The EU eHealth for Safety study [12] anticipates modelling and simulation tools to have a significant impact on patient safety through better prediction, prevention and personalisation.

Firstly, though, we need to accelerate the discovery of new knowledge from large populations of existing health records. Data mining research does not necessarily replace the value of clinical trials, but it can flag up new hypotheses that need further investigation. EHRs can provide population prevalence data and fine grained comorbidity data to optimise a research protocol, and help identify candidates to recruit (almost half of all pharma Phase III trial delays are due to recruitment problems [13]). EHRs are a po- tentially valuable source of outcomes data, if only we could harmonise the information in each EHR and accurately profile each patient to make valid and precise comparisons between fine grained sub-populations. Data warehouses currently support such functions, but are usually limited to a small number of local data feeds (e.g. 3-4 hospitals) and require expensive and time consuming mapping and data cleaning before being usable. We have to get beyond this, to the point where EHRs are populated in the first place with high quality and semantically sound information, and can then be co-analysed consistently to provide a virtual population denominator of millions. Research is still needed, in particular on how to deidentify fine grained clinical data (as opposed to the simple removal of demographic data items) and how to assess and compensate for variable data quality within EHRs.

Secondly, we need to speed up the transfer of newly discovered knowledge into clinical practice. For readily usable clinical knowledge, such as a new therapeutic indication, we know that integrating that knowledge into clinical information systems that are used at the point of care will increase adoption. The more challenging innovations to adopt are radically new ways of thinking, such as molecular medicine and predictive medicine.

Much of molecular medicine focuses on cellular and sub-cellular findings, and maps these to phenotypes. Phenotypes are coarse simplifications of a person's health conditions and health status: they might be suitable to support molecular knowledge discovery but are not usually sufficiently rich to selectively target this knowledge in clinical practice. There is a need for bio-informatics research to integrate more fully with the totality of a patient's clinical picture and clinical course, not just selected phenotypic data items, and therefore to integrate with the EHR. This is vital to support fully personalised medicine. 
Predictive medicine, such as that enabled through the Virtual Physiological Human [14], offers an exciting new way of communicating to clinicians and to patients how subtle changes in a treatment or health condition might impact on body function and eventually outcome. Much of the modelling, though, has been developed in vitro using test data, or clinical data that have been specifically extracted and cleaned. This has been necessary to design the models, but is not the reality of the data on which they will be run in practice: clinical data are full of noise, missing values, and some data entry errors. Research is needed on the tolerance of the models to these noisy conditions, and on ways of measuring and presenting the level of confidence of a prediction.

Just as healthcare has been a cottage industry of parochial standards, so these scientific communities have each standardised within silos, optimising internal sharing but not facilitating integration or collaboration across health and bio-science. Cross-disciplinary semantic interoperability is now needed.

The acceptance of new ways of managing conditions, and of making strategic decisions (such as a chemotherapy agent choice based on bio-markers, or an infusion rate based on cardiac modelling) needs to recognise that clinical decisions are often based on a portfolio of disease/stage/ severity/comorbidity and person/preference/logistic influences. The advanced modelling research communities need to understand better the kinds of diverse inputs that different professionals and specialities have to interpret, the kinds and quality and time spans of data that need to be co-interpreted, the nature and criticality of the decisions being made, and how accurate the modelling projections would have to be in order to be useful. Reciprocally, clinical communities need to understand better what future opportunities and solutions are in the pipeline, how these might impact on care decisions, any adaptations to physical and virtual team-working that should be anticipated and prepared for.

Much of the pure research from bioscience still needs a translational phase in which this applied knowledge can be identified. This additional stage of research is vital if next-generation (3.0) computational services are to make best and safest use of new knowledge to provide sound recommendations to clinicians.

\section{Patient and Citizen Centric Services}

There are growing pressures to recognise the central role of the public as informed partners in decisions about their personal health care, the safety and efficacy of treatment choices and in service priority setting. People are increasingly expecting to exercise personal and informed autonomy over their health care, and we have known for years that individuals can acquire considerable expertise in managing illness and preventive health if they are given useful and appropriate material with which to educate themselves and the tools with which to participate (e.g. [15]). In many countries patients are finally being encouraged (or are themselves pushing) to participate more strongly in the management of their health and healthcare.

However, there is a critical gap in the design and delivery of health care services that fails to harness the immense contributions that patients and citizens can make. These contributions are now vital as healthcare needs and costs make existing models of service unsustainable, and as societal pressure and the growth of health consumerism demands the respect and participation of individuals in healthcare decisions and delivery. There needs to be meaningful sharing of knowledge between clinicians and patients.

A major change is taking place in systems and services to support patients with aspects of self care, mainly realised through Personal Health Record (PHR) systems, which inform and engage patients in health and social care, or enable individuals who are not needing or seeking health care to keep track of their level of fitness, manage prevention or monitor health status. This sector is growing rapidly. Although new entrants to the field (such as Google and Microsoft) offer ways of sending information to clinical systems, there is as yet no real rearchitecting of healthcare services around the patient.

The distinction between a PHR and an EHR is not hard and fast: there will be kinds of shared care records where the distinction is neither clear nor desirable: i.e. the patient and his or her care team might share and manage a common health record transparently. In parallel there is a need for individuals to engage in and take greater ownership of their health and healthcare, including wellness (which existing health services do rather poorly). These are challenges for which the semantic web seems ideally suited: patients and healthy citizens will be able to provide data or pose questions to services that provide intelligent feedback on their data and measurements, perhaps based on simulation models that take account of their past history and present health, medication, lifestyle and environmental factors, and genetic profile. Smart services might provide significant support for self-care, only occasionally escalating a situation to a healthcare professional.

However, the success of patient engagement also critically depends upon attitudes and culture change: policy, organisational, insurance and reimbursement changes, and it has ethical implications. These changes are, in part, needed because patient centred services will include actors whose contributions conventional health services today largely ignore: social care, domiciliary care, health charities, complementary therapists, families etc. In our design of future systems we also need 
to recognise that people are part of families and communities, and do sometimes share and collaborate with others (for example, Patients Like Me [16]). Healthcare and eHealth services probably have a lot to learn from the social social computing paradigm about ways in which people interact, share information and collaborate, and their attitudes to privacy in these contexts.

\section{Trusting Clinical Information}

Investments in interoperability standards, interoperable EHR systems, national eHealth architectures, patient and provider registries and progressively more coherent confidentiality and security policies are making it possible for health record information to genuinely follow the patient between care providers. However, most professionals are used to receiving information from external colleagues in quite formalised ways: reports (e.g. from a lab or radiology department) or letters (referral, discharge, outpatients etc.). Trust in the information provided by colleagues is mixed: there is quite a strong tradition of repeating tests that have just been carried out in other hospitals, re-checking details such as the present and past history with patients, and operating a tacit "hierarchy of evidence" in which more 10cally sourced information is trusted more than information from far away and unfamiliar or overseas organisations. Information provided in less formalised ways (without headed note-paper, undated, unsigned etc.) is usually disregarded.

Professionals are certainly not used to sharing original EHR data on a wide scale: this capability is relatively new. They will need to learn how and when they should trust remote EHR information: its provenance and its certainty. EHR architectures contain properties that capture and communicate some of this provenance information to EHR data recipients. Other aspects of trust are less tangible, and hinge upon the evidence basis for clinical assertions documented within the EHR and the seniority/expertise of the person making the assertions.

Many clinicians still need to learn how to trust patients and citizens with the data they provide, for example in a PHR or (if permitted) into an EHR. The paradox of this is that clinicians have little difficulty in trusting a patient's symptom history (e.g. a diagnosis of migraine, that is almost impossible to confirm except through the history). With the proliferation of increasingly portable, wearable, friendly and connected devices playing vital roles in next-generation tele-care and self-care, this barrier to trust will have to be overcome.

It is less clear still how clinicians will trust information that has been significantly pre-processed by computers, for example a treatment recommendation. The cumulative experience in decision support to date suggests that clinicians are happier to be prompted or alerted about things they might have missed in a health record or in a drug formulary than they are being given "clever" guidance about a clinical judgement. The vision of 3.0 is going to challenge clinicians with information that has been derived from a complexity of information and knowledge sources which they could not practically recreate (and therefore validate) manually i.e. there may be limited transparency. Heath informatics needs to drill into this issue of trust, and seek out ways in which evidence of trustworthiness can be compiled by collaborating services and be available for scrutiny before a recommendation is accepted.

\section{Conclusion}

An integrated information and knowledge environment is not new to healthcare: an old style village GP would have used his memory to inte- grate medical school knowledge and experience with longstanding familiarity with the patient, glancing occasionally at some scrappy paper notes as an aide memoire. Personalised medicine would not be new to him either, since every decision would be made in full awareness of the patient as a person and as a member of a family and community, and a holistic knowledge of all of the patient's health conditions and lifestyle risk factors.

What has changed is the scale, complexity, mobility and quality expectations of modern health care, the rapid pace of change in knowledge, and growing needs for the re-use of health information for many, valuable, nonclinical purposes.

As the data volumes have increased we have elaborated and later outgrown paper records. We are making progress on electronic health records as repositories of data but not yet into shaping the EHR as a knowledge management and knowledge discovery tool. Our notion of shared records is shared access to each of our own electronic records, not yet to collaborate on a systematically organised collective record. We are still cautious about trusting and using information from outside of our own team or institution, and perhaps even more cautious about trusting information entered by patients. We need to progress from integrated electronic health records to collaborative health records: semantically interoperable and supporting virtual teamwork - including the patient's care network.

The pace of medical discoveries and bio-science advances has not only made it impossible to memorise all of what needs to be known (anticipatory learning), but impossible to look up what is relevant on a need to know basis (just in time learning). It is also no longer good enough for knowledge to be incorporated (locked) into specific applications. Facts, evidence, decision logic, care pathway steps, alerts, education need to be embedded within components that can interact with, and thereby serve, multi- 
ple systems and services consistently, and be more easily updated.

Health informatics 2.0 has delivered many significant research, standardisation and implementation building blocks for global eHealth. The mission for Health Informatics 3.0 is to enable citizens, patients and professionals to work together within a knowledge society, informed and advised and empowered by Web 3.0 services in which patient specific information and personalised real-time evidence are seamlessly interwoven.

These are primarily challenges for health informatics to deliver, but we also need to recognise these (positively) disruptive innovations will require acceptance (trust) and adaptation by health professionals and health systems if their benefits are to be fully realised.

\section{References}

1. Berners-Lee T, Hendler J, Lassila O. The Semantic Web - A new form of Web content that is meaningful to computers will unleash a revolution of new possibilities. Scientific American, May 17, 2001

2. Dora L. Costa. The economics and demography of aging. In PNAS - Proceedings of the National Academy of Sciences of the United States of America
(August 14, 2007) Vol. 104 No. 33. p. 13217-8.

3. The UK Collaborative Group for HIV and STI Surveillance. Testing Times. HIV and other Sexually Transmitted Infections in the United Kingdom: 2007. London: Health Protection Agency, Centre for Infections; November 2007.

4. The European Society for Immunodeficiencies registry. Please see http://www.esid.org/ workingparty.php?party $=2 \&$ sub $=2 \&$ id $=233$ [Last accessed 30 April 2011]

5. Stroetman,V., Kalra,D.,Lewalle,P.,Rector,A., Rodrigues,J., Stroetman,K., Surjan,G., Ustun,B., Virtanen,M., Zanstra,P. (2009), Semantic Interoperability for Better health and Safer Healthcare [34 pages]. The European Commission. ISBN-13: 978-92-79-11139-6. DOI: 10.2759/38514. http:// ec.europa.eu/information_society/activities/health/ docs/publications/2009/2009semantic-healthreport.pdf

6. Rogers J, Jain NL, Hayes GM. Evaluation of an implementation of PRODIGY phase two. Proceedings / AMIA Annual Symposium. 1999; 604-8.

7. Kalra D., Editor. The Synapses Object Model and Object Dictionary. EU Telematics Application Programme, Brussels; 1997; The Synapses Project: Deliverable USER 1.3.2. (Copy available from the author on request)

8. Lovis C, Douglas T, Pasche E, Ruch P, Colaert D, Stroetmann K. DebugIT: building a European distributed clinical data mining network to foster the fight against microbial diseases. Stud Health Technol Inform 2009;148:50-9.

9. Krog R, Markwell D, Dolin R, Gabriel D, Cheetham E, Spackman K, et al. Using SNOMED CT in HL7 Version 3; Implementation Guide, Draft Standard for Trial Use. Health Level Seven; 2007.

10. Kalra D, Musen M. ARGOS Policy Brief on
Semantic Interoperability. ARGOS is a Transatlantic Observatory for Meeting Global Health Policy Challenges through ICT-Enabled Solutions. (To be published summer 2011.) Please see http:// www.eurorec.org/RD/ARGOS.cfm

11. Qamar R, Rector A. Semantic Issues in Integrating Data from Different Models to Achieve Data Interoperability. Proc Medinfo 2007 Kuhn K, Warren JR, Leong TY, editors. IOS Press; 2007.

12. Veli N. Stroetmann, Jean-Pierre Thierry, Karl A. Stroetmann, Alexander Dobrev. eHealth for Safety - Impact of ICT on Patient Safety and Risk Management: European Commission. eHealth for Safety Report, October 2007. Luxembourg, Office for Official Publications of the European Communities; 2007.

13. Study participant Recruitment and Retention in Clinical Trials: Emerging strategies in Europe, the US and Asia. Business Insights; June 2007.

14. Please see http://www.vph-noe.eu/

15. Jones R, Pearson J, McGregor S, Cawsey AJ, Barrett A, Craig N, et al. Randomised trial of personalised computer based information for cancer patients. BMJ Nov 1999; 319(7219):1241-7.

16. Please see http://www.patientslikeme.com

\section{Correspondence to:}

Dipak Kalra

Clinical Professor of Health Informatics

Director, Centre for Health Informatics and Multiprofessional Education University College London

Holborn Union Building, Highgate Hill, London N19 5LW Honorary Consultant, The Whittington Hospital NHS Trust, London United Kingdom

Tel: + 442072885966

E-mail: d.kalra@ud..ac.uk 